\title{
INNOVACIÓN: UN PROCESO NECESARIO PARA LAS PEQUEÑAS Y MEDIANAS EMPRESAS DEL MUNICIPIO DE SAN JOSÉ DE CÚCUTA, NORTE DE SANTANDER (COLOMBIA**
}

\author{
William R. Avendaño C.** \\ Recibido: agosto 21 de 2011 • Aceptado: junio 08 de 2012
}

\section{RESUMEN}

El presente artículo tiene por objetivo explorar la necesidad de las Pyme del municipio de Cúcuta en desarrollar actividades encaminadas a la gestión de la innovación, siendo esta uno de los principales instrumentos para el crecimiento de las empresas. Para el logro del objetivo del artículo (de tipo exploratorio y descriptivo), se ha hecho una amplia revisión de diversas fuentes documentales científicas, teóricas y estadísticas. Esta información ha sido extraída, analizada y correlacionada en su totalidad con el fin de dar respuesta al objetivo. Como resultado se resalta la posibilidad que tienen las Pyme para gestionar la innovación, la cual, además de ser una herramienta de desarrollo y crecimiento, genera como efectos otras ventajas económicas y sociales para el municipio de Cúcuta.

\section{PALABRAS CLAVE}

Innovación, pyme, competitividad, Colombia

\section{CLASIFICACIÓN JEL}

\author{
O30, O32, O33, D2 1
}

\section{CONTENIDO}

Introducción; 1. Metodología; 2. Un acercamiento al municipio de Cúcuta y su situación; 3. Condiciones de las pyme en Cúcuta; 4. La innovación: ¿̇una posibilidad de desarrollo para las pyme en Cúcuta?; 5. Beneficios de la aplicación de la innovación en las pyme; 6. Conclusiones; Bibliografía.

\footnotetext{
Artículo de reflexión, producto de los proyectos de investigación desarrollados en el Grupo de Investigación en Responsabilidad Social (GIRS) de la Universidad Francisco de Paula Santander en el marco de la línea de investigación Responsabilidad Social y Empresa.

** Economista en Comercio Exterior, Universidad Los Libertadores, Bogotá, Colombia; especialista en Alta Gerencia, Universidad Libre, Cúcuta, Colombia; magíster en Administración, Universidad Santo Tomas, Bucaramanga, Colombia. Profesor adscrito al Departamento de Estudios Internacionales y de Fronteras, Facultad de Ciencias Empresariales, Universidad Francisco de Paula Santander, Cúcuta, Colombia. Director del Grupo de Investigación GIRS. Correo electrónico: wrac2008@hotmail.com o wrac_ufps@hotmail.com
} 


\section{INNOVATION: A NECESSARY PROCESS FOR SMALL AND MEDIUM COMPANIES OF SAN JOSE DE CUCUTA MUNICIPALITY, NORTE DE SANTANDER (COLOMBIA)}

\section{ABSTRACT}

The goal of this paper is to explore the need that SMEs have in the Colombian municipality of Cucuta in developing activities intended to trigger innovation management, as this one of the main tools for business growth. In order reach the goal of this paper (exploratory and descriptive type), an extensive review was done over several documented sources; scientific, theoretical and statistical. This information has been totally extracted, analyzed and correlated in order to test the results. As a result, the study highlights the possibility that SMEs have for managing innovation, which becomes at the same time a tool for generating growth and development that brings with it other economic and social advantages for the municipality of Cucuta.

\section{KEY WORDS}

Innovation, SMEs, Competitiveness, Colombia

\section{JEL CALSSIFICATION}

O30, O32, O33, D21

\section{CONTENT}

Introduction; 1 . Methodology; 2. A close-up to Cucuta municipality and its current situation; 3. SMEs conditions in Cucuta; 4. Innovation: A development possibility for SMEs in Cucuta?;

5. Benefits of applying innovation in SMEs; Conclusions; Bibliography.

\section{INOVAÇÃO: UM PROCESSO NECESSÁRIO PARA AS PEQUENAS E MEDIANAS EMPRESAS (PeME), DO MUNICIPIO DE SAN JOSÉ DE CÚCUTA, NORTE DE SANTANDER (COLOMBIA)}

\section{RESUMO}

O presente artigo tem como objetivo explorar a necessidade das PeME do município de Cúcuta em desenvolver atividades encaminhadas à gestão da inovação, sendo esta um dos principais instrumentos para o crescimento das empresas. Para conseguir atingir o objetivo do artigo (de tipo exploratório e descritivo), tem se feito uma ampla revisão de diversas fontes documentais científicas, teóricas y estatísticas. Esta informação tem sido extraída, analisada e correlacionada na sua totalidade com o fim de dar resposta ao objetivo. Como resultado se ressalta a possibilidade que tem as PeME para administrar a inovação, a qual, além de ser uma ferramenta de desenvolvimento e crescimento, gera como efeitos outras vantagens económicas e sociais para o município de Cúcuta.

\section{PALAVRAS CHAVES}

Inovação, PeME, Competitividade, Colômbia

\section{CLASSIFICAÇÃO JEL} O30, O32, O33, D2 1

\section{CONTEÚDO}

Introdução; 1. Metodologia; 2. Uma aproximação ao município de Cúcuta e sua situação; 3. Condições das PeMEs em Cúcuta; 4. A inovação: cuma possibilidade de desenvolvimento para as PeMEs em Cúcuta?; 5. Benefícios da aplicação da inovação nas PeME; 6. Conclusões; Bibliografia. 


\section{INTRODUCCIÓN}

Ya desde la década de 1930, Shumpeter (1934) introducía el término innovación y lo asociaba a los experimentos del mercado y a los cambios en los sectores productivos. En efecto, la innovación se concretaba con la introducción de nuevos productos o de métodos de producción, apertura de nuevos mercados, desarrollo de fuentes de suministro de materias primas e insumos y creación de nuevas estructuras de mercado. Lo cierto de estos procesos es el uso del conocimiento como fuente de la innovación (nuevo conocimiento, nuevo uso o combinación de conocimientos existentes) en donde, según OCDE y EUROSTAT (2005, p. 44), este requiere de "esfuerzos innovadores por la empresa que pueden ser diferenciados de las rutinas habituales".

Lo que aquí sobresale es que a pesar del uso de tecnologías, nuevos conocimientos, nuevas aplicaciones del conocimiento, etc., la empresa por sí sola debe ser innovadora, es decir, desarrollar una cultura de la innovación. Por ello explican Escorsa y Valls (2003, p. 21): "las ideas y los conceptos no son innovaciones. Tampoco importa la procedencia de las ideas. Lo importante es que las ideas sean puestas en práctica con éxito para satisfacer a los clientes". Por lo tanto, se requiere un desarrollo de la innovación tanto tecnológica como administrativa si se quiere llegar a una innovación de tipo radical.

La innovación tiene su origen en el desarrollo de nuevas ideas, las cuales sumadas al elemento valor marcan la diferencia con la creatividad como mera capacidad. Lo cierto es que la innovación requiere del conocimiento y de su gestión para alcanzar objetivos y conquistar el mercado. Esta perspectiva se impone, poco a poco, dentro de las organizaciones que habían optado por competir con otros factores como costos, calidad, diseño, plazos y satisfacción al cliente, entre otros. Ya es de conocimiento que estos factores no bastan en los nuevos esquemas socio-económicos impuestos por la globalización y las organizaciones actuales ya que han tenido que modificar su estrategia competitiva. Este nuevo concepto se ha convertido en el paradigma actual de la teoría administrativa desde la visión de Hamel y Breen (2008, p. 14), lo cual rompe con las leyes y principios que prevalecían en la administración. Al respecto describen:

Estas nuevas realidades exigen capacidades organizativas y gerenciales diferentes. Para prosperar en un mundo cada vez más perturbador, las empresas deben poder ser tan estratégicamente adaptables como ya son operativamente eficientes. Para salvaguardar sus márgenes deben convertirse en manantiales de innovación anticonvencional, y para poder ganarle la partida a una multitud creciente de empresas jóvenes en lo que se refiere a ideas e inventiva, tendrán que aprender a inspirar a sus empleados para que den lo mejor de sí mismo día tras día. Estos son los desafíos que los innovadores de la administración tendrán que enfrentar en el siglo XXI.

Citado por OCDE-EUROSTAT (2005, p. 37). 
Resalta de los aportes de Hamel y Breen (2008, p. 7) que el conocimiento tiene una gran influencia sobre la innovación. El conocimiento se acumula, es modificado y reinventado de forma permanente por los innovadores. Se trata de un activo intangible que impulsa diversas posibilidades, reestructura la empresa y les genera valor a los procesos misionales. En la actual sociedad del conocimiento, la información se crea de manera constante y está disponible en las grandes redes facilitadas por las tecnologías de la información y la comunicación, como lo plantea Boisier (2001, p. 11): "(...) la sociedad del conocimiento o la nueva economía tiene un basamiento técnico en las Tecnologías de la Información y de las Comunicaciones (TIC) que empujan la competitividad, la innovación y los cambios organizacionales en y entre empresas". Para Cardona y Cano (2005, p. 2) "(...) las tecnologías [son una] forma de acumulación y difusión del conocimiento" y los trabajadores son verdaderas comunidades de aprendizaje.

Las empresas al innovar no solo introducen al mercado un producto, servicio, concepto, idea y/o proyecto, sino que ofrecen una alternativa llamativa e interesante para la satisfacción de las necesidades de las personas (valor), quienes encuentran en el mercado una gran variedad de ofertas y se convierten en agentes que exigen cambios, piden transformaciones y esperan innovación. Por tal motivo, Méndez (2002, p. 63) afirma que la innovación "se ha convertido, durante la última década, en un concepto de uso cada vez más frecuente en todo tipo de ámbitos, consolidándose la idea de que un esfuerzo de innovación sostenido resulta ser un factor clave para mejorar la competitividad de las empresas y favorecer un desarrollo en los territorios". Y esto es muy relevante si se consideran las nuevas características del mundo globalizado ${ }^{2}$ el cual ofrece un entorno inestable para la empresa (Benavides, 1998, p. 11).

La competitividad resulta ser un objetivo de la innovación. Porter (2009, p. 7) considera la competencia como una de las "fuerzas más poderosas de la sociedad" que permite la creación de valor. Se trata de la capacidad que tiene la empresa o la organización para alcanzar ventajas a través de su posicionamiento en el mercado. Para Porter (2009, p. 17) la "competitividad surge del incremento de la productividad en el uso de recursos" y Porter (2009, p. 32) formula las cinco fuerzas que moldean la competencia: "amenaza de nuevos aspirantes, poder de negociación de los compradores, amenaza de productos o servicios sustitutivos, poder de negociación de los proveedores y rivalidad entre los competidores existentes". Según Porter (2009, p. 543), su perspectiva se basa en el conocimiento pleno del sector y el diseño de estrategias en función de alcanzar ventajas competitivas, y para ello la innovación juega un papel importante: "la innovación conduce

La globalización erige sus bases en la edad media pero los principales hechos que la configuran tienen lugar en gran parte del siglo XX: la profundización de la economía capitalista a partir de la caída del orden social ideológico del anterior siglo en 1989, el desarrollo en el transporte y de las comunicaciones en virtud de los avances en la informática y la electrónica facilitando la transmisión de información, una cultura de consumo y una cultura global. 
Innovación: un proceso necesario para las pequeñas y medianas empresas del municipio de San José de Cúcuta

a la productividad (...) los más importantes avances no surgen del incremento de las mejoras en rendimiento sino de enfoques nuevos más acertados".

La estrategia es clara para la empresa: innovar atendiendo al conocimiento del entorno y a la estrategia de la organización. Es una posibilidad y a la vez un instrumento necesario para competir en la sociedad del conocimiento, la sociedad de la información y la sociedad de las comunidades de aprendizaje. Y no se trata de una herramienta exclusiva para las empresas de gran tamaño, también es adaptable a las más pequeñas, aunque en los estudios, se evidencie su baja capacidad de innovación frente a las empresas de mayor tamaño.

Las pyme ${ }^{3}$ pueden encontrar en la innovación un camino para su proyección, desarrollo y fortalecimiento. Y como describe Hamel (2008, p. 7) no se trata ya de una opción sino de un requisito para la organización, si desea crecer y mantenerse en el mercado (en la curva de la S). El panorama en Colombia es interesante respecto de las pyme, toda vez que representan un amplio porcentaje de la totalidad de las empresas, y a pesar de su flexibilidad para adaptarse al entorno, tiene restricciones de todo tipo; al respecto Torres (2008. p. 112) plantea:

Las pyme y las microempresas constituyen el 99\% de las compañías del país, generan el 63\% del empleo y el 37\% de la producción. Las microempresas son, en su mayor parte, empresas familiares de los estratos 1, 2 y 3. Si bien, en general, su estructura productiva es, más flexible que la de la gran empresa, se identifican importantes restricciones técnicas y competitivas en la medida en que se caractericen por limitadas escalas de producción y por la informalidad.

La importancia de las pyme en Colombia para la economía es significativa y hay consenso en ello. Explica Rodríguez (2003, p. 14) que "las Mypyme son importantes para el desarrollo económico y el equilibrio social en Colombia, por su contribución a la generación de riqueza, empleo, crecimiento económico, bienestar y mejora en la distribución del ingreso".

En el contexto del municipio de Cúcuta, Norte de Santander (Colombia), que por sus características propias, lo hacen especial frente a otras entidades territoriales, la gran mayoría de las empresas se ubican dentro de la categoría Pyme.

El número de las empresas del municipio de Cúcuta (cuadro 1) al año 2011 correspondían a 23.946, de las cuales 22.837 son microempresas (95,36\%), 883 son pequeñas empresas (3,68\%) y 139 son medianas empresas (0,58\%). Solo $88(0,36 \%)$ corresponden a grandes empresas. Es de acotar que para el año 2009 el número

3 De acuerdo con la Ley 590 de 2000 (CR, 2000), artículo 2º la micro, pequeña y mediana empresa, es toda unidad de explotación económica, realizada por persona natural o jurídica, en actividades empresariales, agropecuarias, industriales, comerciales o de servicios, rural o urbana, que cumplen con las siguientes características:

- Micro empresa: Hasta 10 trabajadores y con activos de menos de 501 SMLV

- Pequeña empresa: Entre 11 y 50 trabajadores y con activos desde 501 y menos de 5.001 SMLV

- Mediana empresa: Entre 51 y 200 trabajadores y con activos desde 5.001 y menos de 15.000 SMLV. 
de pequeñas, mediana y gran empresa era superior al del 2009. En este año la composición de las empresas sumaba 22.203, de las cuales 21.002 eran microempresas (94,6\%), 925 eran pequeñas empresas (2,4\%), 153 medianas empresas $(0,7 \%$ y 123 $(0,6 \%)$ correspondían a grandes empresas. Lo que se observa es el incremento de las microempresas y la disminución del número del resto de empresas.

Cuadro 1. Composición micro, pequeña, mediana y grandes empresas en Cúcuta al 2011

\begin{tabular}{|c|c|c|c|c|c|}
\hline Año & Microempresa & Pequeña empresa & $\begin{array}{c}\text { Mediana } \\
\text { empresa }\end{array}$ & Gran empresa & $\begin{array}{c}\text { Total de } \\
\text { empresas }\end{array}$ \\
\hline 2009 & 21.002 & 925 & 153 & 123 & 22.203 \\
\hline 2011 & 22.837 & 883 & 139 & 88 & 23.946 \\
\hline
\end{tabular}

Fuente: elaboración del autor con información de CCC (2012, p. 16)

Así, la economía del municipio de Cúcuta se caracteriza por estar constituida entre pequeños y medianos negocios que pueden asumir la innovación como fuente de cambio y desarrollo, incentivados por la oportunidad que se desprende de la posición geo-estratégica que les sirve de puente con el mercado tradicional de Colombia-Venezuela. De este modo, es necesario partir de una idea base: la economía en Cúcuta se ve traducida en el fenómeno fronterizo que implica un desarrollo de integración previo a cualquier imposición de límites por parte de los Estados. Por tal motivo, existe una gran dinámica en el libre flujo de bienes, servicios y personas (Amorocho: 2002, p. 8) por tratarse de un espacio exclusivo para uso comercial.

$\mathrm{Al}$ atenderse las consideraciones previas tanto teóricas como de contexto, el artículo hace una exploración sobre la necesidad de las pyme del municipio de Cúcuta de aunar esfuerzos en el campo de la innovación, así como los requerimientos para su desarrollo y los beneficios de los que puede ser partícipe un gran sector de las empresas de este espacio fronterizo. Rivillas (s. f.) explica que "en el nuevo escenario mundial la revalorización de lo local como fuente de crecimiento, es decir, la identificación, valoración y proyección de los recursos locales y regionales existentes, pueden significar el impulso a procesos de desarrollo", y en la innovación se puede encontrar un instrumento de alto impacto para lograr estos propósitos y eliminar las barreras que de manera permanente se les presentan a las pyme ${ }^{4}$ : problemas de acceso a los mercados, barreras tecnológicas y dificultades para obtener recursos de crédito del sector financiero (Cárdenas, 2002).

\footnotetext{
Según Cárdenas (2002) este tipo de dificultades no solo afectan a las micro, pequeñas y medianas empresas del país. También se ven limitadas en este mismo sentido las que se encuentran en la Unión Europea y en Estados Unidos.
} 
Del mismo modo, la innovación es una posibilidad para generar mayores niveles de competitividad donde la productividad aumente en la medida que se reduce el trabajo informal, el cual se encontraba en el 65,9\% para el 2011 mostrando una tendencia de aumento. En efecto, el rendimiento de un trabajador formal es siete veces mayor al de un trabajador informal (Cámara de Comercio de Cúcuta, 2012. p. 23).

El artículo expone, en una primera parte, la situación socio-económica del municipio con el análisis de algunos indicadores suministrados por fuentes oficiales, luego se hace una exploración de las condiciones de las Pyme en la ciudad y se exponen las posibilidades de innovación como fuente necesaria para el desarrollo de las pyme. Finalmente, se presentan las conclusiones del trabajo realizado.

\section{METODOLOGÍA}

El objetivo del artículo es explorar la necesidad que tienen las pyme del municipio de Cúcuta de desarrollar actividades en torno a la gestión de la innovación, ya que esta herramienta es importante para su crecimiento y posicionamiento. Se trata de un artículo exploratorio por cuanto "el objetivo es examinar un tema (...) poco estudiado o que no ha sido abordado antes" (Hernández, Fernández y Baptista, 2000, p. 58), en otras palabras, se hace una exploración lo que corresponde al "primer nivel de conocimiento científico" (Méndez, 2004, p. 134); y descriptivo toda vez que se "ocupa de las características que identifican los diferentes elementos, componentes, y su interrelación" (Méndez, 2004, p. 136). Frente a los estudios descriptivos Hernández, Fernández y Baptista (2000, p. 61) añaden que estos "miden independientemente los conceptos o variables a los que se refieren". Para el cumplimiento del objetivo se acude a diversas fuentes científicas: teoría, informes estadísticos y documentos de diversa índole, de la cual se extrae la información necesaria para análisis e interpretación a los largo del artículo

\section{UN ACERCAMIENTO AL MUNICIPIO DE CÚCUTA Y SU SITUACIÓN}

A continuación se presenta una corta revisión de aspectos socio-económicos del municipio de Cúcuta (ubicación fronteriza, relaciones binacionales, balanza comercial, mercado laboral, inflación, mercado internacional) a fin de comprender sus fortalezas y limitaciones y poderlas analizar posteriormente con las pyme y la innovación como herramienta estratégica.

San José de Cúcuta, con una población de 776.000 habitantes, es una ciudad ubicada en el nororiente colombiano (región andina), capital del departamento Norte de Santander y espacio primordial en el comercio de Colombia y Venezuela. Sin embargo, esta característica no le fue propia a la ciudad en un principio, ya que en la época de la colonia tuvo funciones político-administrativas. Ya para la segunda mitad del siglo XIX la ciudad se proyectaba como un espacio moderno, comercial y 
cosmopolita, aún más, después de la reconstrucción del municipio producto del terremoto de 1875. Durante este período, la ciudad desarrolló su capacidad económica y financiera a causa de la interacción con el vecino país lo cual trae como efectos la consolidación de esas cualidades que la hacen tan especial. Para Iturriza (1986, p. 166) las áreas de frontera son economías regionales aisladas de los centros dinámicos y de las decisiones nacionales, con ausencia de políticas específicas para su promoción, que aprovechan las oportunidades que marcan las diferencias económicas entre las dos naciones. Esto es claro en la realidad histórica de Cúcuta, cuyo crecimiento estuvo influenciado por la bonanza petrolera de Venezuela en las décadas de 1970 y 1980.

Gómez, Linares y Bradshaw (2005, p. 6) advierten que debido a la naturaleza cambiante de las funciones fronterizas, los espacios limítrofes han pasado de una perspectiva de conflicto y hostilidad a representar cooperación y armonía "particularmente dentro de los procesos de globalización y de cooperación transfronteriza". La ubicación geográfica de Cúcuta es una ventaja para su economía y, sin embargo, los niveles de competitividad son bajos y con una relativa especialización (Bustamante y Bustamante, 2008. p. 22).

Cúcuta ha sido considerada como una ciudad comercial por excelencia, el primer puerto terrestre de Colombia, con un nivel de desarrollo que ha dependido de las políticas económicas de ambos países más que de potencialidades propias (Amorocho, 2002), aunque estas no hayan tenido una visión compartida y pertinente. Este conjunto de limitaciones también las expresa el informe del BID-INTAL-CAF (citado por Amorocho 2002, p. 285):

Para esas colectividades vecinas, la consolidación del proceso de integración global, en lo que respecta al caso colombo-venezolano, los diversos sectores sociales, económicos y políticos requieren la creación de condiciones económicas, jurídicas e institucionales que les permitan tener una presencia y participación más activa en el devenir de las sociedades y las economías a las cuales pertenecen.

Es menester observar que la historia de esta frontera se encuentra enmarcada por altibajos, avances y retrocesos como lo afirman Ramírez, Romero y San Juan (2005), para quienes las limitaciones de esta zona responden a los cambios recientes en las políticas exteriores y en particular en la posición frente a Estados Unidos, pero sobre todo, por el aumento de las divergencias políticas que han hecho resurgir y aumentar los temores mutuos.

Entre Colombia y Venezuela se han presentado varios inconvenientes que han perjudicado no solo a los grandes empresarios del país sino también a la comunidad del municipio de Cúcuta. Durante la pasada crisis, generada por la denuncia del Acuerdo de Cartagena por parte del Gobierno de Venezuela y las diferencias entre ambos gobiernos, la Dirección de Impuestos y Aduanas Nacionales (DIAN) y el 
Departamento Administrativo Nacional de Estadísticas (DANE), registraron para el año 2010 la caída de las exportaciones en un 65\%, lo que representó casi el 4\% del total exportado por Colombia al mundo. Para el año 2011 (14 de mayo) la situación no había variado significativamente. Solo se presentó el 1\% de incremento (USD578 millones) respecto del mismo período en el 2010 (USD 574 millones). Sin embargo, a partir de este período y en abril del 2011 se observa una nueva tendencia hacia el crecimiento de las exportaciones (19\%) que significó USD 116 millones, es decir, USD 18 millones más respecto del mismo período del año anterior. En cuanto a las importaciones de Colombia respecto de Venezuela, habían registrado una caída del 45\% y para mayo del 2011 se incrementaron las importaciones en un 102\% y en abril en un $257 \%$.

Con la nueva administración del presidente Juan Manuel Santos, se ha reactivado el comercio con Venezuela, lo que colabora con la situación de Cúcuta. La ciudad fronteriza debió tomar medidas fuertes al momento en que la crisis tocó fondo pero las cifras infieren normalidad en las relaciones comerciales sin desconocer los efectos que deja: inseguridad jurídica, ampliación de la oferta exportable, consolidación de nuevos mercados internacionales, etc. Según la CCC (2011, p. 11), a finales del 2011, los datos registraron que el principal mercado internacional de Cúcuta lo seguía constituyendo Venezuela (27\%), seguido por Estados Unidos (14\%), Brasil (8\%), Panamá (7\%), Indica (7\%), China (6\%), Bélgica (5\%), Argentina (4\%), Finlandia (3\%), Turquía (2\%), Suecia (2\%), Ecuador (2\%) y Noruega (2\%).

El mercado laboral del municipio de Cúcuta y su área metropolitana, según la CCC (2011, p. 4), está caracterizado por tener 603.000 personas en edad de trabajo es decir, $77,7 \%$ de la población en total del área metropolitana. La población activa es de 401.000 personas y de estos, 340.000 personas se encuentran ocupadas (86.7\%) y 60.000 desocupadas (13,3\%). La tasa de desempleo al 2011 fue de 18,7\% (Cámara de Comercio de Cúcuta, 2011, p. 4). Pero es preciso aclarar que de las 340.000 personas ocupadas 224.000 son informales mientras que 116.000 son formales, ocupando el municipio el segundo lugar en informalidad en Colombia. Las 224.000 personas laboralmente activas no reciben como producto de su trabajo las prestaciones sociales necesarias que el orden jurídico exige garantizarles. Esto en materia de promover el mínimo vital es una problemática de orden social y un obstáculo para el desarrollo de la competitividad y la innovación. Son cifras elevadas que infieren una dificultad propia de la coyuntura económica del municipio para la generación de estrategias de innovación y, por ende, consolidación de la competitividad y el crecimiento.

Según la CCC (2011, p. 9), la inflación del municipio al 2011 cerró con una tasa del 4,64\% y el índice nacional fue de 3,29\% (Cámara de Comercio de Cúcuta, 2011, p. 9). El principal factor de esta situación fue el invierno, que afectó las vías y el 
transporte, elementos esenciales en la economía de una zona fronteriza como Cúcuta. Además, este indicador refleja que Cúcuta, en el ámbito nacional, es una de las ciudades con más alto índice en el costo de vida, afectando la capacidad de compra de los consumidores.

\section{CONDICIONES DE LAS PYME EN CÚCUTA}

Cúcuta, hacia la década de 1970, fortaleció su posición como principal puerto de exportación de bienes colombianos hacia Venezuela. La ciudad se conformó, además, como un importante centro comercial y de servicios, dotada de un gran dinamismo, conferido por las compras de alimentos, textiles y otros bienes de consumo final, realizadas por venezolanos. Las ventajas comparativas de los precios de diversas manufacturas colombianas contribuyeron de manera decisiva para que se mantuviera el auge comercial de Cúcuta durante esa década.

La ubicación fronteriza de Cúcuta y Villa del Rosario sirvió para que se convirtieran en fuentes permanentes de mano de obra para el sector industrial venezolano, como sitio de residencia transitoria o semipermanente para aquellos colombianos y otros migrantes andinos que aspiraban ingresar a Venezuela legal o ilegalmente. En el eje San Antonio del Táchira-Ureña, se incrementó en esa década el establecimiento de pequeñas y medianas industrias, en particular en la zona de Ureña.

La localización de inversiones en la zona fue posible, entre otras razones, por los costos ventajosos de la mano de obra y de algunas materias primas de origen colombiano, así como por la existencia de una importante oferta de trabajadores colombianos que en ese momento encontraban dificultades para su inserción en los mercados laborales de Colombia. De igual forma, San Antonio del Táchira, fortalecía su posición como centro comercial, basado en un considerable prestigio en el ámbito binacional en lo referente a precios competitivos en artículos como productos electrónicos y electrodomésticos importados de otros países.

Las peculiares condiciones económicas, sociales, tecnológicas, políticas y geográficas de las pyme de Cúcuta son, grosso modo, conocidas por quienes en esta región fronteriza residen o tienen afinidad. Para nadie resulta ser un secreto la precaria situación que se vive como consecuencia del desarrollo de una cultura comercial que depende de la variabilidad de cotización de las monedas de Colombia y de Venezuela. Las políticas concretas del gobierno nacional y, de manera absurda, las acciones de los entes del orden departamental y municipal, a ciencia cierta, no han apoyado la industria regional, situación que hoy contribuye en el proceso de desintegración económica de las pyme del municipio. A éste panorama crítico se unen las deficientes capacidades administrativas, financieras y competitivas de las empresas, así como la baja formación del talento humano y el escaso nivel tecno- 
lógico. Por ello, las empresas de Cúcuta han sido vulnerables frente a los diferentes procesos económicos y de integración, como la apertura económica, la globalización, y ahora ni qué pensar frente a los Tratados de Libre Comercio, lo que conduce a pensar en un colapso económico inminente. Esto es evidente al observarse que la cantidad de micro empresas aumentó de 2009 al 2011 y las pequeñas y medianas empresas disminuyeron (ver cuadro 1).

La mayoría de las pyme del municipio manejan una dirección orientada hacia el tradicional gerenciamiento paternalista y administración empirista, que conduce a un accionar equívoco desde el punto de vista competitivo, teniendo en cuenta, la falta de planeación estratégica de la empresa. Según Rodríguez (2003, p. 18), la mayoría de las pyme del municipio son de carácter familiar y en un $50 \%$ son administradas por los mismos propietarios, quienes tienen en un gran porcentaje estudios profesionales (56\%) y un nivel más bajo (16\%) tienen estudios de postgrado. El 70\% de los empresarios de este tipo de organizaciones tienen estudios superiores. Otras características que refleja el estudio de Rodríguez (2003, p. 18) respecto del perfil del gerente de la pyme en Colombia, es que tiene un gran conocimiento del negocio y dentro de las funciones que desempeña se encuentran las de administrar (64\%), vender (16\%), gerencia o representación legal (7\%), producción y contabilidad (6\%). De acuerdo con Rodríguez (2003, p. 18), los gerentes, en su mayoría los mismos propietarios, se dedican a administrar la empresa pero "las habilidades de gestión se encuentran limitadas". Prima la ausencia de gerentes con formación en gestión, donde si bien tienen conocimiento del negocio, prima el empirismo, y su lógica marca su accionar, carente de planificación (costos, oportunidad, estrategia, etc.) e infraestructura física y logística que soporten su sostenibilidad y crecimiento. De allí que requiera de procesos de innovación y todo lo que ello implica.

Argumenta Rodríguez (2003, p. 23), que otros elementos categorizados como obstáculos que deben atenderse para las pyme y que son percibidos por los propietarios y gerentes de estas empresas son: la situación económica del país, el sistema tributario, el orden público, el poco apoyo del Estado, el acceso a la financiación, la legislación laboral y el mercado interno. Un dato que resulta relevante es que un alto porcentaje de gerentes o propietarios de las pyme no consideran el mercado externo como una limitante, ya que no tienen intención de explorarlo. Esta última perspectiva refleja una limitante de tipo cultural que obstaculiza el desarrollo, el crecimiento y la competitividad de la pyme en Cúcuta. Las causas las describe Rodríguez (2003, p. 83) en tres vertientes: "complejidad por la tramitología para exportar, deficiencias en información y mercadeo y dificultades en el proceso de exportación".

Las microempresas, con todas estas dificultades externas e internas, han sido el motor productivo y generador de empleo más representativo en Cúcuta, lo cual contribuye de manera significativa en la generación de fuentes de empleo en el 
sector comercial. En la zona de frontera de Cúcuta y su área metropolitana se manejan diversas políticas comerciales, sociales y laborales que desembocan en una compleja problemática debido a los cambios económicos que se promueven entre Colombia y Venezuela, y al abandono de la frontera por parte del poder central. De todas formas, la innovación sigue siendo una opción para las pyme.

Otra posición necesaria de abordar es que la innovación no es propia de las grandes empresas. Es posible desarrollarla en cualquier plano, en diferentes ámbitos, micro, pequeñas y medianas empresas. Ubeda y Moslares (2008, p. 27) plantean que la innovación ha estado presente hoy y siempre, constituida como un pilar fundamental de la actividad humana, que explica el éxito empresarial como algo que no se le puede atribuir exclusivamente a las empresas de mayor tamaño, pues sería auto-limitar la capacidad de las pyme en su crecimiento.

\section{LA INNOVACIÓN: ¿UNA POSIBILIDAD DE DESARROLLO PARA LAS PYME EN CÚCUTA?}

La innovación es fundamental e imprescindible en el nuevo esquema global. No es permitido pensar en el desarrollo y el avance sin la promoción de la innovación en los diferentes niveles de la organización sin importar el tipo de empresa. Sin embargo, el concepto de innovación se ha manifestado en diversas perspectivas a través de una transformación histórica. Formichela (2005, p. 40) observa que:

(...) el concepto ha variado a lo largo del tiempo, volviéndose cada vez más amplio. En un principio sólo se consideraban dentro del mismo las innovaciones en productos, luego se incorporaron las innovaciones en servicios, hasta llegar a la inclusión de las innovaciones en procesos y las organizativas.

De la misma forma, plantea un cambio de paradigma entre las décadas 1970 y 1980, en donde la innovación pasó a ser de exclusividad del sector público, a definirse y promocionarse como prioridad de las empresas privadas. Para Malaver y Vargas (2004, p. 35) se ha y se está dando una "creciente importancia de la innovación como determinante primordial de la competitividad en el actual entorno económico".

La innovación, que proviene del latín innovatio, es definida por la Real Academia de la Lengua Española en dos sentidos: a) como acción y efecto de innovar y, b) como creación o modificación de un producto, y su introducción en el mercado. Innovar, por su parte, se define como a) mudar o alterar algo, introduciendo novedades, y b) volver algo a su anterior estado. Lo que se observa de estas definiciones aportadas por la Real Academia es el cambio como elemento fundamental. En efecto, términos como creación, modificación, mudar, alterar o volver evocan la noción que se tiene de transformación o cambio que en todo caso conduce a la mejora. Otros dos aspectos relevantes son el producto y el mercado, y esto tiene coherencia con lo que 
plantean Jiménez y Sanz (2006, p. 33) para quienes la innovación se clasifica según su naturaleza en tecnológica o administrativa, es decir, que con ellas se responde a las necesidades de los clientes y usuarios. El concepto innovación ha variado atendiendo a las perspectivas y al contexto propio de las sociedades, y es por ello que es imposible unificar criterios en función de homogeneizar una definición entre los diversos autores. Sin embargo, existe una amplia literatura alrededor del tema de la innovación que resulta de perentorio análisis.

Drucker (2002, p. 95) identifica la innovación como una "herramienta específica de los empresarios" que permite generar cambios y explotar oportunidades de negocio para crear un valor económico que, hasta el momento, no ha existido. Para Dávila, Epstein y Shelton (2005, p. 12) también significa una oportunidad de impacto en el mercado. Entonces, la creación de valor económico así como el impacto en el mercado son dos funciones primordiales de la innovación. En este orden, lo anterior no significa que otros aspectos no sean importantes pues bien como describe Afuah (1999, p. 20) los estudios económicos, financieros y de mercado, así como la teoría de la organización, son importantes para la gestión de la innovación. En términos de Jiménez y Sanz (2006, p. 33) citando a Afuah (1999, p. 20) la innovación "se basa en el conocimiento organizativo existente".

La innovación debe basarse en las relaciones que se tejen entre las empresas y las necesidades de los usuarios (Lundvall, 2009, p. 10) sin descuidar la "estructura económica, las instituciones y las políticas para el aprendizaje". Por la misma línea expone Rosenau (1988, p. 3, 24, 43, 59, 77, 99, 113, 139) la idea de innovación, para quien resultan trascendentales la estrategia, el mercado, el producto, el plan de beneficios y el trabajo en equipo, es decir, elementos organizativos y tecnológicos en el marco de las necesidades y la satisfacción de los clientes. Este autor imprime una gran importancia a los dos primeros elementos: estrategia y mercado.

Precisamente el mercado resulta ser un factor esencial en la innovación, pues es en este espacio, donde se busca generar impactos significativos a partir de los nuevos o mejorados productos o los cambios gestados en la organización. Conforme a la profundidad de las transformaciones provocadas, las innovaciones pueden clasificarse en incrementales o radicales. Para Jiménez y Sanz (2006, p. 33) la primeras mantienen cambios menores y son organizacionales mientras que las segundas incluye mayores cambios y son de tipo tecnológico. Hidalgo, León y Pavón (2002, p. 63) describen que las innovaciones incrementales se caracterizan por el conocimiento y la previsibilidad de la demanda, la rápida aceptación del mercado, la fácil adaptación a las ventajas del mercado y a la política de distribución, y por encajar en la segmentación del mercado; mientras que la innovación radical tiene un riesgo elevado, tiene poco conocimiento de la demanda en el mercado, no es previsible la reacción de la competencia, exige 
políticas de mercadeo, distribución y ventas, y puede ser que no coincida con el segmento del mercado. Las innovaciones incrementales asumen poco riesgo en comparación con las innovaciones radicales, pero del mismo modo, su impacto no es tan profundo y es muy poco probable que orientan el comportamiento del mercado a partir de los cambios generados. En contraposición, las innovaciones radicales tienen una alta posibilidad de fracasar ya que el riesgo es muy alto, el mercado desconocido y la reacción de los consumidores inesperada.

Con el fin de limitar los aportes conceptuales de los diferentes autores en el siglo XXI alrededor del tema de la innovación, resulta acertada la recopilación que hacen Jiménez y Sanz (2006, p. 33-34) y la de otros autores consultados. En el cuadro 1 se sintetiza el tratamiento conceptual dado al término innovación.

Cuadro 2. Definiciones de innovación

\begin{tabular}{|c|c|}
\hline Autor(es) & Conceptualización \\
\hline $\begin{array}{l}\text { Shumpeter (1934) citado por } \\
\text { Jiménez y Sanz (2006, p. 33, 34) }\end{array}$ & $\begin{array}{l}\text { Introducción de un nuevo bien o método, cambio en la calidad, apertura } \\
\text { de un nuevo mercado, creación de una nueva organización. }\end{array}$ \\
\hline $\begin{array}{l}\text { Sherman (1981) citado por } \\
\text { Escorsa y Valls (2003, p. 20) }\end{array}$ & $\begin{array}{l}\text { Proceso en el cual a partir de una idea, invención o reconocimiento de } \\
\text { una necesidad se desarrolla un producto, técnica o servicio }\end{array}$ \\
\hline $\begin{array}{l}\text { Pavon y Goodman (1981) citado } \\
\text { por Escorsa y Valls (2003, p. 20) }\end{array}$ & $\begin{array}{l}\text { Conjunto de actividades inscritas en un determinado periodo de tiempo y } \\
\text { lugar, que conducen a la introducción con éxito en el mercado de produc- } \\
\text { tos nuevos o mejorados, servicios o técnicas de gestión y organización }\end{array}$ \\
\hline $\begin{array}{l}\text { Tushman y Nadler (1986) citado } \\
\text { por Jiménez y Sanz (2006, p. } \\
\text { 33, 34) }\end{array}$ & Creación de un producto, servicio o proceso. \\
\hline $\begin{array}{l}\text { Piatier (1987) citado por Escor- } \\
\text { sa y Valls }(2003, \text { p. 20) }\end{array}$ & Idea transformada en algo vendido o usado. \\
\hline Davenport (1996, p. 10) & $\begin{array}{l}\text { Introducción de algo nuevo con el propósito de generar un cambio } \\
\text { grande, radical. }\end{array}$ \\
\hline $\begin{array}{l}\text { Damanpour y Gopalakrishnan } \\
\text { (1998) citado por Jiménez y } \\
\text { Sanz }(2006, \text { p. 33, 34) }\end{array}$ & Adopción de una idea; comportamiento nuevo en una organización. \\
\hline Escorsa y Valls $(2003$, p. 20) & $\begin{array}{l}\text { Idea nueva hecha realidad o llevada a la práctica; explotación con éxito } \\
\text { de nuevas ideas. }\end{array}$ \\
\hline $\begin{array}{l}\text { OCDE y EUROSTAT (2005, p. } \\
\text { 36) }\end{array}$ & $\begin{array}{l}\text { Introducción en el mercado de un producto o proceso nuevo o significa- } \\
\text { tivamente mejorado o el desarrollo de nuevas técnicas de organización } \\
\text { y comercialización. }\end{array}$ \\
\hline
\end{tabular}

Fuente: Elaboración propia a partir de Jiménez y Sanz (2006, p. 33-34), Escorsa y Valls (2003, p. 20), OCDE y EUROSTAT (2005), Danvenport (1996, p. 10) 
Ahora bien, la innovación ha demostrado ser esencial para todas las organizaciones sin importar características, tamaño o alcance. Frente a esta concepción, y de acuerdo con la posición que se mantiene en el artículo, Lébre y Hasenclever (2003, p. 1) explican que la constitución de la sociedad del conocimiento puso de manifiesto el papel de las pyme en el nuevo paradigma tecno-económico puesto que atributos como flexibilidad y capacidad innovadora presentes en las pyme pasaron a ser vistos como esenciales para la competitividad.

Analizado y comprendido que la innovación es factible en las empresas más pequeñas, como las que realizan su actividad en el municipio de Cúcuta, es perentoria la revisión del entorno con el fin de determinar sus características. En cuanto al actual paradigma tecno-económico, según Lébre y Hasenclever (2003) los factores determinantes del contexto son:

a. Fin del régimen de sustitución de importaciones en la mayor parte de los países en desarrollo y adopción de varias reformas orientadas al mercado que modificaron de forma trágica las condiciones de competencia en el mercado interno;

b. Globalización de las actividades económicas, caracterizada por el crecimiento de flujos internacionales de comercio, tecnología y capital, así como una creciente interconexión de los sistemas productivos, redefinición de las relaciones entre firmas y regionalización de los espacios nacionales con la formación de bloques de comercio;

c. Transición para sociedades basadas en el conocimiento, esto es, sociedades basadas en la transmisión y uso del conocimiento y de la información, donde la capacidad innovadora de las firmas en su proceso competitivo desempeña un nuevo papel, y caracterizadas por un fuerte crecimiento del trabajo cognitivo y relacional; $\mathrm{y}$

d. Cambio técnico y organizacional con la expansión del ohnismo o del toyotismo ${ }^{5}$ y el surgimiento de un paradigma tecnológico basado en la microelectrónica, nuevos materiales y biotecnología.

En cuanto a esta última característica del paradigma tecno-económico, Peirano y Suárez (2005, p. 14) establecen en su investigación que existe una relación entre las TIC y la innovación como elemento fundamental para mantener la competitividad:

En efecto, las actividades de innovación que se realizan en contextos donde se han establecido estas redes o sistemas de intercambio e interacción se ven influenciadas positivamente, lo cual permite esperar mayores y mejores resultados. Las

\footnotetext{
Para Díaz, García y González (1997), es un modelo de producción que nace en Japón y que se basa "en la detección de la demanda y la producción del bien en función de una necesidad específica"; en otras palabras, la producción y la oferta se modifican de acuerdo a la demanda y sus características.
} 
actividades de I+D pueden ahora nutrirse de un abundante caudal de información técnica disponible en Internet o que circula por las redes interfirma.

En síntesis, las empresas del municipio de Cúcuta pueden encontrar en la innovación una herramienta que amplíe las posibilidades de desarrollo de la ciudad. La innovación no constituye un proceso complejo al que sólo pueden acceder las grandes empresas. De esta manera, se desmitifica la idea sesgada que la innovación no es compatible con las empresas más pequeñas. Por el contrario, las características que les son propias a las pequeñas empresas permiten, por su flexibilidad y capacidad de cambio, generar nuevos esquemas innovadores. Sin embargo, aún se reconocen muchas limitantes a este tipo de empresas que, no obstante, pueden ser superadas mediante acciones pertinentes y con visión de los gobiernos (local, departamental y nacional), de acuerdo con la importancia económica que representa para los intereses del país. Es necesario, por ende, un cambio de perspectiva que revista de la real importancia a esta zona de frontera olvidada.

\section{BENEFICIOS DE LA APLICACIÓN DE LA INNOVACIÓN PARA LAS PYME}

Existe un consenso entre los autores acerca de los beneficios para las empresas a partir de la aplicación de estrategias innovadoras, inversión en tecnología y sobre los procesos de I + D (Benavides y Quintana, 2002. p. 19; Aboites y Soria, 1999). Algunos de los beneficios de esta herramienta para las pyme del municipio de Cúcuta son:

a. Crea una relación armoniosa entre la organización y el cliente. Este se convierte en el principal beneficio al momento de innovar, toda vez que la satisfacción del cliente se ve elevada por motivo del cubrimiento de necesidades. Asimismo, el primer principio de la calidad es la satisfacción al cliente, lo cual es posible si: a) se investiga para comprender acerca de las necesidades del cliente, b) se formula y se asegura la dirección estratégica de forma correcta, c) se desarrolla un plan de vinculación general entre los miembros de toda la organización y c) se mide de manera constante la satisfacción de los clientes y se gestiona de acuerdo con esos resultados.

b. Aumenta el volumen de ventas. Al satisfacer las necesidades de los clientes aumenta su número, al igual que las ventas lo que conduce a la conquista de nuevos mercados o sectores nunca antes explorados.

c. Mejora la imagen de la organización. La innovación mejora la imagen, gracias a la generación de nuevas expectativas promovidas por los requerimientos de la llamada sociedad del conocimiento. Con ello, se fortalece el posicionamiento de la organización. 
d. Disminución de costos. Una característica de la innovación es generar o diseñar procesos que sean más eficientes y eficaces, es decir, que se puedan alcanzar los objetivos propuestos mediante un mejor uso de los recursos. A esto se le puede llamar reducción de costos mediante la efectividad de los procesos diseñados.

Son múltiples los beneficios que se pueden lograr mediante la innovación. Se han nombrado los cuatro principales y a través de ellos, hay muchos que se pueden adquirir, como el aumento de la rentabilidad a partir de la disminución de costos y el aumento de volumen de ventas o el mejoramiento de la calidad y el desarrollo de la cadena de valor, entre otros.

\section{CONCLUSIONES}

La innovación no es un tema nuevo y de acuerdo con lo expuesto por diversos expertos, tiene una relación estrecha con el marco económico y el aprovechamiento de las oportunidades que genera el medio. Del mismo modo, la innovación es el resultado de un cambio inevitable que se ha iniciado por la búsqueda y construcción de una nueva teoría o paradigma de la administración en reemplazo de las viejas formas, que siendo aún aplicables merecen ser reestructuradas, ya que la globalización impacta la configuración económica, social, política y cultural que se tiene del mundo, afirmando que la innovación es la respuesta a una sociedad globalizada cuyos cambios exigen transformaciones constantes que resultan eficaces para los cambios requeridos por las Pyme en su lucha por sobrevivir a los grandes cambios y exigencias de mercados que impone la globalización y la nueva tendencia económica.

De acuerdo con la información recolectada, analizada e interpretada en las diversas fuentes, se ha podido llegar a las siguientes conclusiones:

Cúcuta a pesar de su larga historia como zona comercial del país y de actividad mercantil marcada, se encuentra estancada y esto se debe al escaso nivel de competitividad e innovación propio de la región. Cúcuta es una ciudad con ventajas comparativas que pueden convertirse en ventajas competitivas aprovechando su característica de zona fronteriza. La vecindad del municipio con el Estado venezolano, principal mercado internacional para Cúcuta y Colombia, constituye una ventaja.

Los elementos macroeconómicos que caracterizan al municipio de Cúcuta son obstáculos en el desarrollo de estrategias innovadoras para las empresas. Una de las principales barreras para la implementación de la innovación como instrumento de las pyme es la informalidad laboral.

Las condiciones políticas derivadas de las relaciones entre Colombia y Venezuela son una oportunidad para el desarrollo y crecimiento de las pyme en el municipio de Cúcuta. 
La innovación conduciría al crecimiento de las pyme en el municipio de Cúcuta y a la reducción de las diferencias sociales, la informalidad laboral y la mejora de las condiciones laborales. Las pyme, al ser flexibles, como producto de su propia naturaleza, pueden aprovechar la innovación de una mejor forma. Resulta ser una herramienta para mejorar su productividad, su organización, tecnificarse, responder mejor al mercado, aprovechar el conocimiento de sus trabajadores y dirigir el mercado marcando pautas de consumo.

Es prioritario para las pyme conocer de manera amplia el negocio, el mercado y sus tendencias, y el entorno propio del municipio para generar verdaderas propuestas de innovación.

Debe ser incluida dentro de la estrategia de las pyme, la exportación como actividad potencial a mediano y largo plazo. Es fundamental en un proyecto que intenta generar innovación y competitividad regional, la participación dinámica y permanente de diversos actores: gobierno, empresa, universidad, sociedad civil, instituciones públicas. La innovación es una posibilidad para las pyme por su flexibilidad, porque es aplicable y no se excluye, ya que ofrece relevancia a la actividad del trabajador como recurso intangible de la organización.

Son múltiples beneficios a los que puede acceder las pyme con la aplicación actividades tendentes a gestionar la innovación: mayor productividad, aumento en las ventas, acercamiento con el cliente, posicionamiento en el mercado, crecimiento y desarrollo, expansión del negocio, creación de una cultura innovadora permanente, creación de imagen, disminución de costos, aprovechamiento de recursos.

\section{BIBLIOGRAFÍA}

Aboites, J. y Soria, M. (1999). Innovación, propiedad intelectual y estrategias tecnologicas: la experiencia de la economía mexicana. México: UAM.

Afuah, Allan (1999). La dinámica de la innovación organizacional: el nuevo concepto para lograr ventajas competitivas y rentabilidad. México: Oxford University, 496p.

Amorocho Pérez, Amanda Patricia (2002). Política social y desarrollo en la zona urbana fronteriza de la ciudad de San José de Cúcuta, Colombia, p. 282-297. En: Álvarez Raquel, Giacalone Rita y Sandoval Juan Manuel (Comp) (2002); Globalización, integración y Fronteras en América latina. Mérida: Gratemica, 326p.

Benavides Velasco, Carlos Ángel (1998). Tecnología, innovación y empresa. Madrid: Pirámide, 364p.

Benavides Velasco, Carlos Ángel y Quintana García, Cristina (2002); Regiones en aprendizaje. ¿Una nueva dimensión territorial de la innovación? En: Boletín Económico de ICE, No. 2722, p. 19-30. 
Innovación: un proceso necesario para las pequeñas y medianas empresas del municipio de San José de Cúcuta

Bid-Intal-Caf (1997). Programa de Desarrollo Integral Fronterizo Colombo-venezolano. Área Táchira - Norte de Santander, julio.

Boisier, Sergio (2001). Sociedad del conocimiento, conocimiento social y gestión territorial. En: Interações, Vol. 2, No. 3, p. 9-28.

Bustamante, Ana Marleny y Bustamante, Juan Carlos (2008). Análisis de percepciones sobre la competitividad territorial de tres municipios fronterizos de Norte de Santander y Táchira. En: Aldea Mundo, Año 13, No. 25, p. 13-24.

Cámara de Comercio de Cúcuta (2011). Análisis económico regional 2011. Cámara de Comercio de Cúcuta, Cúcuta, diciembre de 2011.

Cámara de Comercio de Cúcuta (2012). Informe de Gestión. Cámara de Comercio de Cúcuta, Cúcuta, enero de 2012. http://www.cccucuta.org.co/media/Archivos _ Generales/informe_de_gestion_2011.pdf. [6 de junio de 2012].

Cárdenas Santa María, Patricia (2002). Las Pymes: vitales para la recuperación del crecimiento económico. Pymes exportadoras de Colombia, Universidad Sergio Arboleda. http://www. usergioarboleda.edu.co/pymes/noticia8.htm. [19 de junio del 2011].

Cardona Acevedo, Marleny y Cano Gamboa, Carlos Andrés (2005). Innovación empresarial y capacidades tecnoproductivas: análisis sectorial y regional en Colombia en el período 1990-2002. Grupo de Estudios sectoriales y territoriales (ESYT) de la Universidad EAFIT, Medellín. http://ip-50-62-12-119.ip.secureserver.net/documentos/conferencias/medellin/2006/ InnovacionEmpresarial.pdf [4 de junio de 2012].

Danvenport, Thomas H. (1996). Innovación de procesos. Madrid: Díaz de Santos, 344p.

Davila, T., Epstein, M. y Shelton, R. (2005). Making Innovation Work. Upper Saddle River: Wharton School Publishing.

Díaz, Marcela; García, Ruth y González, Alejandra (1997). ¿Orden o Desorden? Una lectura del mundo contemporáneo. Buenos Aires: Signo, 87p.

Drucker, Peter (2002). The discipline of innovation [La disciplina de la innovación]. En: Harvard Business Review, Vol. 63, p. 95-103.

Escorsa Castells, Pere y Valls Pasola, Jaume (2003). Tecnología e innovación en la empresa. Barcelona: UPC, 325p.

Formichela, María Marta (2005). La evolución del concepto de Innovación y su relación con el Desarrollo. Monografía realizada en el marco de la Beca de Iniciación del INTA: Gestión del emprendimiento y la innovación. Estación Experimental Agropecuaria Integrada Barrow, Tres Arroyos, Argentina. http://www.fidamerica.cl/admin/docdescargas/centrodoc/centrodoc_1142.pdf [25 de junio del 2011]

Gómez, Heriberto; Linares de Gómez, Rosalba y Bradshaw, Roy (2005). El paisaje fronterizo: una visión satelital Caso San Antonio del Táchira (Venezuela) - Cúcuta (Colombia). En: Aldea Mundo, Año 004, No. 008, p. 5-18.

Hamel, Gary y Breen, Bill (2008). El futuro de la administración. Bogotá: Norma, 376p. 
Hernández Sampieri, Roberto; Fernández Collado, Carlos y Baptista Lucio, Pilar (2000). Metodología de la investigación. México: McGraw Hill, 501p.

Hidalgo Nuchera, A., León Serrano, G. y Pavón Morote, J. (2002). La gestión de la innovación y la tecnología en las organizaciones. Madrid: Pirámide, 559p.

Iturriza, Jorge (1986). Integración Fronteriza. Un enfoque Metodológico. En: Revista Integración Latinoamericana, No. 118, p. 165-180.

Jiménez Jiménez, D. y Sanz Valle, R. (2006). Innovación, aprendizaje organizativo y resultados empresariales. Un estudio empírico. En: Cuadernos de economía y dirección de la empresa, No. 29, p. 31-55.

La Rovere, Renata y Hasenclever, Lía (2003). Innovación, competitividad y adopción de tecnologías de la información y de la comunicación en pequeñas y medianas empresas: algunos estudios de caso sobre Brasil. En: Boscherini, F., Novivk, M. y Yoguel, G. (Comp) (2003); Nuevas tecnologías de información y comunicación. Los límites en la economía del conocimiento. Buenos Aires: Miño y Davila, 300p.

Lèbre La Rovere, Renata y Hasenclever, Lía (2003). Innovación, competitividad y adopción de tecnologías de la información y de la comunicación en pequeñas y medianas empresas: algunos estudios de caso sobre Brasil. Instituto de Economía, Universidad Federal de Río de Janeiro, Brasil. http://www.littec.ungs.edu.ar/eventos/renata\%20la\%20rovere\%20y\%20 lia\%20hasenclever.pdf. [28 de mayo de 2012].

Lundvall, Bengt-Åke (2009). Innovation as an Interactive Process: User-Producer Interaction to the National System of Innovation. En: African Journal of Science, Technology, Innovation and Development, Vol. 1, No. 2 y 3, p. 10-34.

Malaver Rodríguez, Florentino y Vargas Pérez, Marisela (2004). El comportamiento innovador en la industria colombiana: una exploración de sus recientes cambios. En: Cuadernos de Administración, Vol. 17, No. 27, p. 33-61.

Méndez A., Carlos E. (2004). Metodología. Diseño y desarrollo del proceso de investigación. Bogotá: McGraw Hill, 246p.

Méndez, Ricardo (2002). Innovación y desarrollo territorial: algunos debates teóricos recientes. En: Revista Latinoamericana de Estudios Urbano Regionales. Vol. 28, No. 84, p. 63-84.

Organización de Cooperación y Desarrollo Económicos (OCDE) y Oficina de Estadísticas de las Comunidades Europeas (EUROSTAT) (2005). Manual de Oslo. Guía para la recogida e interpretación de datos sobre innovación. Madrid: Tragsa, 188p.

Peirano, Fernando y Suarez, Diana (2005). Las TIC mejoran el desempeño de las pymes. ¿Somos capaces de explicar cómo lo hacen?, Centro de Estudio sobre Ciencia, Desarrollo y Educación Superior, Argentina. http://unq.academia.edu/FernandoPeirano/Papers/387200/ Las_TICs_Mejoran_El_Desempeno_De_Las_PyMEs._Somos_Capaces _ De _ Explicar_Como_Lo_Hacen. [29 de junio del 2011].

Porter, Michael E. (2009). Ser competitivo. Boston: Harvard Business School, 627p.

Ramírez, Socorro; Romero, Carlos y San Juan, Ana María (2005). Estados Unidos - Colombia Venezuela: ¿una relación triangular? En: Ramírez, Socorro y Cárdenas, José María (2005); 
Innovación: un proceso necesario para las pequeñas y medianas empresas del municipio de San José de Cúcuta

Venezuela y Colombia: Debates de la historia y retos del presente. Caracas: Universidad Nacional de Colombia -IEPRI- y Universidad Central de Venezuela (UCV), 293p.

Rodríguez, Astrid Genoveva (2003). La realidad de la Pyme colombiana. Desafío para el desarrollo. Bogotá: Fundes, 186p.

Rosenau, Milton D. (1988). La innovación. La gerencia en el desarrollo de nuevos productos. Bogotá: Legis, 252p.

Rivillas Castriñon, Diana Marcela (s.f.). Pymes: Los grandes aportes de los pequeños. http://www. seminariopublica.info/maindata/seminario/200824-195535/imagesdirs/Ponencia _ 9 _ RivillasCastrillonDM.pdf [18 de junio del 2011].

Schumpeter, J. (1934). The theory of economic development. Cambridge: Harvard University Press

Torres Salamanca, Raúl (2008). Reflexiones sobre las pymes y el funcionamiento económico nacional. Ecos, No. 2, p. 111-121.

Ubeda Sales, Ricardo y Moslares García, Carlos (2008). Innovando la innovación. En: Boletín Económico de Información Comercial Española (ICE), No. 2942, p. 27-38. 